\title{
Developing Trust In Virtual Software Development Teams
}

\section{Valentine Casey ${ }^{1}$}

\author{
${ }^{1}$ Dundalk Institute of Technology, Department of Computing and Mathematics, val.casey@dkit.ie
}

Received 19 February 2010; received in revised form 18 June 2010; accepted 20 June 2010

\begin{abstract}
Today globally distributed software development has become the norm for many organizations and the popularity of implementing such an approach continues to increase. In these circumstances a strategy often employed is the use of virtual software development teams. Due to the collaborative nature of software development this has proved a difficult and complex endeavor. Research has identified distance in its various forms as an important factor which negatively impacts on global software development and on virtual software team operation in particular. In this context, the aspects of distance have been defined as temporal, geographical, cultural and linguistic. A key element for the success of any team based project is the development of trust and cooperation. Each aspect of distance can negatively impact on the development of trust and hamper cooperation particularly in the virtual team environment. An additional factor which this research identified is the importance and negative impact fear plays. The serious implications of these factors are due to the need for dependence on asynchronous and online communication which is inherent to global software development and the operation of virtual software teams in particular. The findings presented here are the results from four independent studies undertaken over a twelve year period which consider each of these issues. Having identified the problems associated with trust and communication, how these issues were successfully addressed and managed on a multimillion dollar project which was heading for failure is outlined.
\end{abstract}

Key words: Trust, Fear, Cooperation, Online Communication, Global Software Development (GSD), Virtual Teams, Grounded Theory, Action Research 


\section{Introduction}

Globally distributed software development has become a popular strategy for many organizations to implement and the popularity of this approach continues to increase [50], [59]. This has been made possible by the availability of qualified software engineers, located in low cost economies that are capable of undertaking outsourced and offshored software development [74]. This has also been facilitated by the revolution in communication which the Internet has made possible. This has resulted in the use of online tools which include email, the World Wide Web, Instant Messenger, Skype, video conferencing and bespoke Internet based communication and operational tools. As a consequence, there has been a substantial reduction in the cost of international communication [58]. Taking these factors into consideration the rationale for the adoption of a Global Software Development (GSD) strategy has been attributed to organizations endeavoring to gain and maintain competitive advantage [63], [70]. It is believed this can be achieved by capitalizing on the perceived advantage of labor arbitrage between geographical locations [6]. This can then be coupled with leveraging the temporal difference between these locations which can provide opportunities for reduced time to market and facilitate competitive pricing. Increasingly organizations are endeavoring to leverage these opportunities to enable them to establish, maintain and / or expand their market share in what are often volatile international markets [22], [38].

This has resulted in software development becoming a globally sourced commodity [37] which has facilitated the migration of software development and maintenance operations to geographically distributed locations. In some circumstances maintenance and application development have been outsourced to third party organizations or partners. In others, organizations have established subsidiaries in low cost economies and offshored software development projects to these locations. While both of these strategies have become popular, it should be note that due to the level of complexity which is inherent to software development, outsourcing or offshoring have both proved they can be difficult endeavors [41]. Some of the problems encountered include factors such as understanding requirements, testing and the coordination of these types of projects [74]. These difficulties have been compounded by factors such as cultural and linguistic differences, communication problems and geographical and temporal distance. Different process maturity levels, development and testing tools, standards and the technical ability and experience of staff. As a result the operation and management of GSD projects is now recognized as a difficult and complex tasks [8], [26], [31].

Distance has been identified as a key issue and its potential to negatively impact on GSD projects should not be under estimated. By its very nature distance introduces barriers and complexity into the management of globally distributed projects [66] see figure 1.

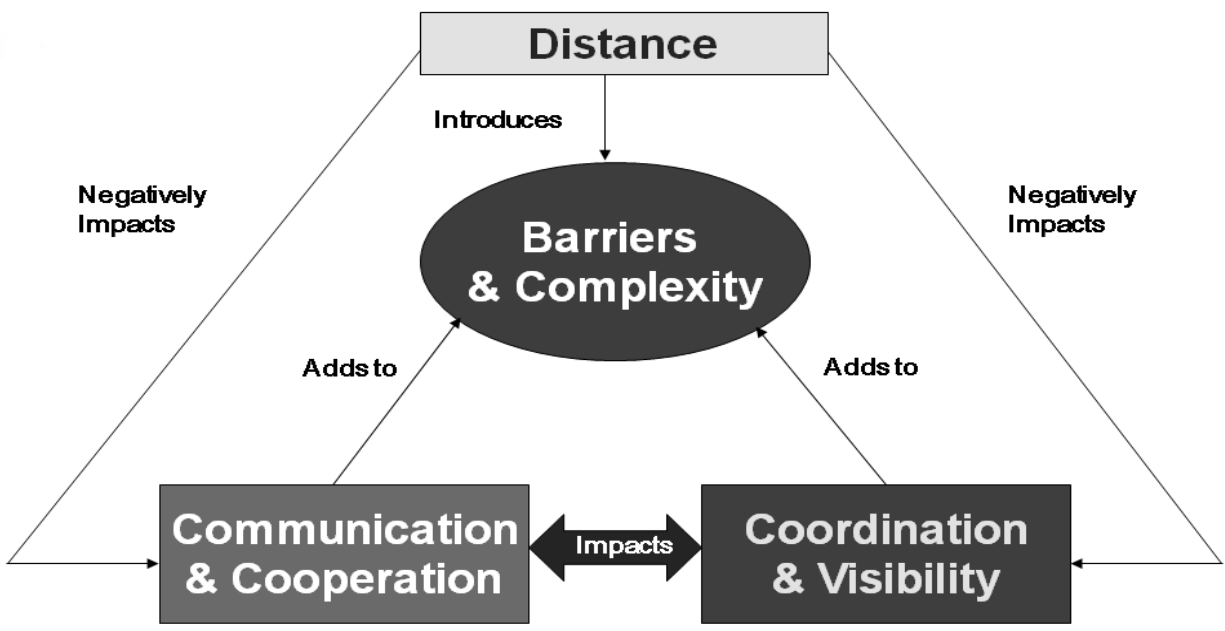

Figure 1: The Impact of Distance when Operating in a GSD Environment [16]

This can be directly attributed to the fact that in the GSD context there are four key elements to distance. Geographical distance introduces physical separation between team members and management [15]. Temporal distance hinders and limits opportunities for direct contact, cooperation and the development of trust [5], [63]. Linguistic distance limits the ability for coherent communication to take place [45]. Cultural distance negatively impacts on the level of understanding and appreciation of the activities and efforts of remote colleagues and teams [37]. As a result of distance, communication on GSD projects is normally electronic with limited opportunities for synchronous contact, depending on geographical location and time zone difference. Coordination, visibility, communication and cooperation are all negatively impacted by geographical, temporal, linguistic and cultural distance [14]. If these important elements of distance and their potential for negatively impacting on projects are not 
recognized and managed correctly they can be responsible for creating serious barriers and complexity within GSD projects [20].

\subsection{Virtual Software Teams}

A popular approach when implementing a GSD outsourcing or offshoring strategy is the establishment of virtual teams. The virtual team has been described as the core building block of the virtual organization [25]. A traditional team has been described as a social group of individuals who are collocated and interdependent in their tasks. They undertake and coordinate their activities to achieve common goals and objectives and share responsibility for outcomes [62]. Virtual teams have the same goals and objectives as collocated teams, but operate across, geographical locations, time zones and are linked by communication technologies [52]. A virtual team has been formally defined as a team whose members use the Internet, intranets, extranets and other networks to communicate, coordinate and collaborate with each other on tasks and projects even though they work in different geographical locations [58]. The virtual team differs from a traditional collocated team in that its members are distributed across geographical sites, but remain interdependent in their tasks [52]. Virtual teams also normally operate in a multicultural and multilingual environment, which often cross divisional or organizational boundaries [28].

\subsection{Focus of this Work}

The focus of this work is the development of trust in the virtual software team environment. Professional software development is complex and normally team based. It is therefore important to recognize it is not just a technical activity. It also has important social, collaborative and knowledge sharing aspects which are key to its success [48]. In the past this fact has often been overlooked or trivialized. A key factor which is essential for the development of effective social, collaborative and knowledge sharing relationships is trust [42]. This is the case in collocated software teams and is even more important when operating in a virtual team environment [56]. As Handy (1995) states "Virtuality requires trust to make it work: Technology on its own is not enough" [35]. In these circumstances it is relevant to note that the development of trust in a virtual team can be a difficult and complex endeavor [4].

The virtual environment and distance both negatively impact on the development of trust [9]. The loss of face to face contact hinders the development of relationships between virtual team members [16]. The requirement to utilize asynchronous communication tools limits contact and knowledge sharing [15]. This is often compounded by team members in different locations having difficulty communicating due to their knowledge of English and / or the use of different dialects of the language [20]. Cultural differences can also lead to misunderstandings and misrepresentation of the actions and activities of remote colleagues [8]. Each of these factors can negatively impact on the development of trust between virtual software team members.

A major issue which until recently was not fully appreciated in the context of globally distributed software development is the negative impact that fear can have on the effective operation of virtual teams [61]. This is particularly relevant with regard to the development and maintenance of trust [19]. To be effective virtual teams must operate in a cooperative and collaborative manner. Fear has been identified as a key inhibitor for the establishment and operation of cohesive and motivated virtual teams [15]. These problems have often been exacerbated in organizations utilizing what has been termed the "cost and productivity mantra" [17]. This is where management inform virtual team members located at the offshoring or outsourcing location that their remote colleagues cost a lot less than they do. As a result they should therefore be more productive and value adding [14]. The objective of utilizing this approach is twofold, one to maintain or reduce costs in the outsourcing or offshoring location and two to motivate staff and increase productivity. On both counts, as the results from the studies presented in this paper clearly demonstrate, this approach has failed and negatively impacted on the operation of the respective virtual teams and projects [20]. Fear limits or prevents communication, collaboration, knowledge sharing and hinders cooperation which all negatively impact on the development of professional relationships and trust between virtual team members. Having identified the problems associated with building trust in the virtual software team environment, it is important to consider how these problems can be successfully addressed.

The research, on which this work is based, has been undertaken over a twelve year period. The first study took place from 1998 to 2000 and the second study took place from 2001 to 2003 . The third study was undertaken over a four year period from 2003 to 2007 . The fourth study began in 2007 and having produced a number of results, is ongoing. Given the longitudinal nature of this research, it is relevant to note that similar results are still being identified by others, who are investigating this area [59].

The key point of this paper is to highlight the important role trust plays in the successful operation of virtual software teams. In this context it is also important that the factors which hinder the development of trust are identified, understood and addressed. These issues need to be considered at the team level which is discussed in section 7 . They also need to be tackled at the organizational level, to this end a structured approach to the establishment and operation of virtual software teams has been developed [18], [20]. In addition, based on the results which have been achieved from these studies, research is underway since 2009 on how these findings can be incorporated into measures to address these issues from an industry wide perspective. Both of these topics are considered in section 8. 


\section{Research Studies}

As outlined in section 1 the findings presented in this work are based on the results from four independent studies undertaken over a twelve year period. Virtual software teams were selected as the basis for this research given their level of exposure to the full implications of operating in a globally distributed environment. In this context it is recognized that the findings which are presented here have relevance not only to the operation of virtual software teams, but also to the other types of GSD offshoring and outsourcing strategies which can be employed [45].

The four studies are classified under the GSD strategy headings outlined by Hayes [37]:

1. Local offsite software development: Virtual team members were both part of the same organization and were dispersed over two locations one hundred and fifty miles apart, in the same country.

2. Offshore / nearshore software development: Virtual team members were located in the United States and Ireland. The project was a partnership between a US based financial organization and the Irish division of a US multinational. The sites were geographically distant, but they were considered linguistically and culturally nearshore.

3. Offshore software testing: Virtual team members were geographically, linguistically and culturally distant, with members based in Ireland and Malaysia. In this study both sites where divisions of the same US multinational organization. The Irish division had been the recipients of offshored projects from their US parent for the previous twenty years and they were now responsible for offshoring part of their projects to Malaysia.

4. Offshore software development: This study is focused on offshore software development and virtual team operation. There is a specific focus on the factors and management issues that are particularly relevant for the recipients of this type of work. To achieve this objective research has been undertaken with three Indian based software development organizations. This study has delivered some results and is still ongoing.

\subsection{Local Offsite Software Development}

The goal of the first study was to research the operation and what turned out to be the subsequent failure of a local offsite virtual software development team strategy [7]. This strategy had been implemented subsequent to the takeover by a large Irish owned software organization Irish Computing Solutions (a pseudonym) of a small software company based in Dublin (the capital). The company, which had been acquired, had a proven track record in the financial and telecommunication software sectors. Having experienced some initial problems after the acquisition, these issues were promptly identified and addressed. As a result the operation was successfully managed for two years and integrated as a separate unit into Irish Computing Solutions. At that point it was decided that a new strategy should be implemented. This was to expand and develop the organization's market share as a whole by the establishment of local offsite virtual software development teams. Irish Computing Solutions had a software development center located 150 miles from Dublin which had lower labor costs than the capital. The objective was to leverage both locations and capitalize on the cost advantage which this strategy could provide.

Twelve offsite engineers were selected and provided with basic training in the technology and process with the goal of being partnered with six experienced Dublin based onsite engineers. After the training period two virtual teams were established and consisted of two sets of six offsite engineers who were partnered with three experienced onsite engineers. Considerable effort was put into providing the communication infrastructure, process and support for both virtual teams. A key objective of this approach was that the onsite engineers would mentor the inexperienced offsite staff and provide effective knowledge transfer. In reality, this did not take place. The experience of the offsite engineers can best be summed up in the following quote: “The onsite engineers won't tell us anything, they won't even return our calls." The majority of communication which took place was restricted to email and these were limited in the information they provided or were not always answered. This took place even though a number of different management strategies including a process improvement initiative were implemented to facilitate communication and cooperation between team members at both locations [7]. Given these circumstances within a period of six months of its full implementation the strategy had to be abandoned due to its total failure.

\subsection{Offshore I Nearshore Software Development}

The second study focused on what is termed offshore / nearshore software development. The concept of offshore / nearshore was derived from the fact that the research centered on a partnership between a US based financial organization Stock Exchange Trading Inc. and the Irish division of a US multinational - Software Future Technologies (both pseudonyms). Ireland though geographically offshore, is often considered nearshore, because of its linguistic and cultural similarities to the US [37], [68]. The partnership between these two organizations resulted in the establishment of virtual teams to develop and maintain bespoke financial software. Stock Exchange Trading Inc. was the senior partner in this relationship. 
Previously all Stock Exchange Trading Inc. software development and maintenance activities were undertaken inhouse in the US. As a result of continued expansion the organization identified an increasing demand for software development and maintenance. They also realized that their in-house strategy had become too expensive. The solution was to find an efficient alternative, which would leverage the experience of their existing software development department while maintaining the level of quality and support required at a cost effective price.

Stock Exchange Trading Inc. had previously successfully outsourced their Y2K legacy renovation to an Irish based division of Software Future Technologies. The possibility of building on this relationship was identified and explored. After extensive negotiations a four-year contract was agreed and signed. The terms of this contract outlined that both companies would partner and establish virtual teams to undertake the development and maintenance of all Stock Exchange Trading's software applications.

After the selection and training of the Irish based team members, an unanticipated demand arose, which required seventy percent of the Irish team members to spend six to twelve months working onsite in the US in Stock Exchange Trading Inc. This unanticipated need arose from the requirement for the development of complex bespoke software within a short time period to meet the needs of a recently acquired subsidiary. When this demand was identified the virtual teams were in an early ramp up phase and the infrastructure for their operation was not complete. The solution was moving as many Irish team members as possible to work on site with their US based team colleagues for a temporary period. This proved to be an effective strategy and both groups operated very successfully while collocated within what were to eventually become their virtual teams. When the urgent projects were completed the Irish team members returned to Ireland and the virtual teams were established.

Initially everything went well, but soon serious problems were encountered with the operation of the virtual teams. An online communications war commenced between the US and Irish based virtual team members. Rather than utilizing email as a communication tool it was used primarily as a weapon to attack colleagues in the other location. This was initiated by the US based team members utilizing email to highlight any minor problems that arose with their colleagues in Ireland [13]. The Irish staff responded by adopting a similar negative approach. As a result this became normal practice in the day to day operation of the teams. This activity had the desired effect of highlighting minor problems, but it alienated the individuals it was directed toward. It also had a negative impact on their fellow team members at that geographical location, who saw it as an attack on their group as a whole [14].

This situation was further compounded by management reacting to these emails and getting involved with issues that the relevant team leaders should have addressed. It was noted that the management in their response normally took the side of individuals where they were located. This negative approach directly led to the alienation of staff and management at both locations based on geographical and organizational lines [14]. The result was a breakdown in cross-site relationships, effective communication, trust and cooperation. It is of particular relevance to note these problems arose despite the fact the individuals involved had worked very successfully together for over a year when collocated. The severity of these problems negatively impacted on productivity and resulted in increased project overruns in time and costs. Within a short period of time this threatened the partnership between both organizations and urgent action was required [13]. Once the most urgent problems were identified and measures put in place to address them. The study considered why these issues had arisen and how to address the root causes of these problems. It then went on to investigate how best to leverage the advantages that the virtual team strategy could provide and this was successfully achieved.

\subsection{Offshore Software Testing}

The third study focused on offshore virtual team based software testing [9] and took place over a three year period. The study was undertaken in the Irish division of a US multinational, Computing World Inc. (a pseudonym) which had been operating in Ireland for over twenty years. Over that period, a large percentage of the projects undertaken were offshored from their US parent; therefore, the Irish staff and management were very familiar with having projects offshored to them. Two years prior to undertaking this study the Irish division of Computing World Inc. initiated a new strategy of establishing virtual testing teams with members based in Ireland and their Malaysian division. The objective of undertaking this approach was to leverage the technical ability of the Irish based staff with the competitive salary levels of the Malaysian engineers.

When this research commenced four virtual testing teams were in operation with the Irish based division. Two teams were established for over a year and a half while the other two had been in operation for a number of months. This study centered on two embedded units of analysis. One was a virtual testing team with members located in Ireland and Malaysia which had been in operation for a period of eighteen months. The second was a virtual team with a similar makeup, but had been established for six months. Each team specialized in testing specific software for different technologies.

Computing World Inc. supported this research and provided the opportunity for extensive on site access which was successfully leveraged. The implementation of a Yin [76] based case study allowed the preparation and structures to be put in place to maximize the opportunity which the high level of onsite access provided. The inductive 
sophisticated grounded theory [71] based data collection and analysis approach, facilitated the emergence of the relevant factors and issues which directly impacted on the activities of the virtual team members at both locations.

\subsection{Offshore Software Development}

Having previously carried out research, which considered the perspective of remote project managers and staff who had been the recipients of outsourced software projects, in the Irish context [9], [13]. The relevance of undertaking broader research in this area was appreciated. This was confirmed by a review of the relevant GSD literature. The outcome of which, highlighted that a large proportion of the published literature on globally distributed software development had centered on the requirements of Western organizations outsourcing or offshoring their software development activities. In these circumstances it was considered of value to undertake research which focused on the perspective of the recipients of this work in a broader geographical context. In particular it was considered pertinent to focus on the perspective and experience of remote managers.

Research was undertaken with senior personnel from two large Indian based software organizations and one small Indian software company. An inductive grounded theory based approach was employed [72]. The study comprised of five telephone interviews of one hour duration. The participants included a vice-president, chief consultant, two senior project managers and a project manager. It was recognized that the interviews had to be leveraged to ensure that the best use was made of each opportunity and the respondent's time. Given the inductive nature of the approach employed the objective at all times was to allow the respondents articulate what they considered relevant and to expand on these issues.

Interviews were conducted in both English and Hindi. The choice of language was left to the manager being interviewed and in the majority of cases they chose to mix the two. Flexibility in this area was considered important as it allowed the interviewees to express themselves in whichever language they felt most comfortable. Each interview was recorded with the interviewee's permission. These were then transcribed and translated into English where necessary. They were then analyzed in detail and the findings were identified and verified with the respondents. The results which emerged from the analysis of the data focused on the specific issues which were relevant for the establishment and operation of the relevant Indian based GSD teams [11]. This is an ongoing study and further research and analysis has taken place which has confirmed and expanded on the initial published findings [29], [30]

\section{Methodologies}

The selection and implementation of a relevant research methodology is a key aspect of any research based investigation. For each of the studies outlined considerable time and effort was employed to ensure the most appropriate methodology was selected and employed. In both the local offsite and offshore / nearshore software development studies the alternatives were evaluated. After due consideration the action research five-phase cyclical process based approach as defined by Susman and Evered [73] and Baskerville [1] was selected for both studies.

This methodology was deemed appropriate for both studies as the author held the position of software quality manager with Irish Computing Solutions, when the local offsite study was undertaken. There were similar circumstances with the offshore / nearshore software development study which commenced three years later with Software Future Technologies. As action research entails the analysis of the direct intervention of the researcher [33], the objective in both studies was to leverage the research opportunities which this provided while maintaining the required level of objectivity.

\subsection{Action Research}

Action research is a variant of quasi-experiment research. The difference between action research and other methods of research is the collaborative nature of the relationship between the 'client' (the organization where the research is undertaken) and the researcher. Agreement between both is required at each milestone of the study [33]. This level of collaboration ensures acceptance of the definition of the problems and solutions proposed.

Action research consists of a five-phase cyclical process based approach as defined by Susman and Evered [73] and outlined in [1]-[2] The five-phases are executed within the boundaries of the client system infrastructure and are:

- Diagnosing

- Action Planning

- $\quad$ Action Taking 
- Evaluation

- $\quad$ Specify Learning

The implementation of participatory action research [2], [32] was of particular value. The bounded results based iterative approach afforded by the implementation of the action research strategy provided effective results for both the local offsite and offshore / nearshore software development studies.

\subsection{Yin Based Case Study}

The offshore software testing study required a different approach and research methodology than the previous two studies. When this investigation was undertaken the author was a fulltime researcher and was offered the opportunity to undertake extensive onsite research. The objective was to maximize the opportunities which this level of access facilitated. Having researched and considered the alternatives the requirement for a qualitative, structured, but inductive approach was identified. This resulted in the selection of a Yin [76] based embedded case study which incorporated a Strauss and Corbin grounded theory [72] based inductive approach to data gathering and analysis. A Yin based case study is normally considered a positivist qualitative research approach [47]. Therefore it was only selected for this study when it was clearly evident that it could be legitimately implemented utilizing an exploratory and inductive data gathering and analysis approach.

Empirical studies all have an implicit or explicit research design. Research design is defined by Yin (1994) as "an action plan for getting from here to there". The term here is described as the initial research questions and there as the conclusions reached. Central to the design of an efficient research plan is the requirement to put structures in place. The rationale for doing this is to ensure that the research remains focused on its fundamental goals and objectives. The focus of the offshore software testing study was that it should be carried out in an ideographic manner, which embarked on an exploratory journey of discovery that remained bounded in its relevant context while not being constrained by it.

When developing a research design for undertaking case study based research Yin [76] identifies five relevant components that should be addressed:

- The study's questions

- Its propositions, if any

- $\quad$ The unit(s) of analysis

- The logic linking the data to the postpositions

- The criteria for interpreting the findings

These were utilized as the basis for the research design for the offshore software testing study. Of particular relevance was the identification of the propositions.

Each proposition is defined as a mechanism for directing the researcher's attention to specific areas that should be focused on within the scope of a case study. This approach allows the researcher to develop theories prior to undertaking research as to what the possible outcomes of the investigation might be. Given the exploratory and inductive nature of the approach implemented in this study it was clear propositions were not applicable. Therefore it was very important to ensure this was an appropriate approach to employ. It was identified that Yin recognized that when undertaking exploratory case study based research there is a legitimate reason for not having propositions and he states:

"At the same time some studies may have a legitimate reason for not having any propositions... in which a topic is a subject of "exploration" [76].

There was a requirement to consider what would determine the success of the investigation for the offshore software testing study. As a result the purpose and overall objectives of the research needed to be focused on and success determined by the achievement of these objectives [76]. This approach was not preemptive as to what the findings might be. Rather it was broad enough to consider what factors could constitute success.

In a similar way the logic linking the data to the propositions and the criteria for interpreting the findings, were related. Both components were linked to the data analysis stage of the case study. As a result of the exploratory nature of the strategy implemented in the offshore software testing study, the findings had to emerge from the data. Rather than linking the emergent data to the propositions there was a requirement to link the data to the overall purpose and objectives of the research. 
The research design was successfully utilized as the basis for the development of a case study protocol for this study. The protocol provided an overview of the research objectives. It identified the sources of data and briefly outlined the procedures for gathering information. It detailed the broad research questions as defined in the research design and also outlined the proposed analysis method. Yin (1994) states that a research protocol increase the reliability of case study based investigation and should be used as a guide to undertaking this type of research. The case study protocol was presented and agreed with the management of Computing World Inc.

Having agreed the case study protocol the next step was the identification of the scope of the units of analysis. It was determined that a single case study was the most effective method to employ. The rationale for selecting this approach was that it offered the opportunity to study what Yin (1994) terms a revelatory case. Yin goes on to point out the vulnerability of a single case study which must be reflected on, to minimize the possibility of misrepresentation. There needed to be ample opportunity to access and collect the necessary case study data. When both these areas had been satisfactorily addressed then an effective single case study strategy could be embarked on. As a result it was determined the single case study would incorporate two embedded subunits of analysis. These equated to the operation of two virtual teams within the testing department. These two subunits of analysis were representative of the testing department as a whole and were the primary focus for this study [9].

\subsection{Grounded Theory - Data Gathering and Analysis}

To cater for the exploratory and inductive nature of the offshore software testing and offshore software development studies the implementation of grounded theory was selected as the most appropriate strategy for data gathering and analysis. The primary goal in implementing a grounded theory based strategy for both of these studies was to fully leverage the opportunities they provided. The author had prior professional experience and had previously carried out research in the area of virtual team operation, as outlined. He also had extensive knowledge of the GSD literature. In these circumstances for both studies it was important to facilitate the development of theories grounded in the data which they produced. It was also important to acknowledge previous experience and knowledge, but to ensure this did not impede or hinder the finding from either study. In these circumstances it was considered necessary to implement the key characteristics of a sophisticated grounded theory approach as outlined by Strauss and Corbin (1998) [72]. These include:

- The ability to step back and critically analyze situations

- The ability to recognize the tendency toward bias

- The ability to think abstractly

- $\quad$ The ability to be flexible and open to helpful criticism

- Sensitivity to the work and actions of respondents

- A sense of absorption and devotion to the work process

In terms of grounded theory, data analysis is characterized by microanalysis which is a line-by-line review of data. This is undertaken with the objective of identifying initial concepts and defining their properties and dimensions. It is also utilized to identify relationships between these concepts. A key aspect of microanalysis is coding. Coding is defined as the process by which data is fractured, conceptualized and integrated to form theory [72]. The objective is to define, through questioning and theoretical comparison, the concepts and categories within the data and to determine their properties and dimensions. The overall goal is to achieve a better understanding of the data through a combination of open and axial coding.

Open coding is utilized to uncover, name and develop concepts and categories within the data. This process is termed conceptualizing. A concept is defined as significant phenomena, which has been identified and labeled as such in the data. Each concept has recognizable characteristics and dimensions, which allow it to be abstractly defined and coded. In this manner, data is broken down into discrete categories based on similarities and differences [72].

Axial coding is a method employed to add depth and structure to the categories that have been identified in the data. This can provide insight into the structures that created the circumstances which facilitate the existence of such categories. The objective is to reassemble the data in a meaningful way to gain a better understanding of the phenomena and their internal and external relationships. Open coding and axial coding are not necessarily sequential events. Rather, they can be iterative activities which require numerous re-evaluations of the data as the analysis continues [71]. 
The practice of writing memos is a key element in the open and axial iterative coding process. In the context of Grounded Theory a memo is defined as: "The researcher's record of analysis, thoughts, interpretations and directions for further data collection" [72]. All these practices were employed in both studies. When the results emerged from the analysis of the data for both studies they were triangulated with the existing literature in the areas of virtual team operation, GSD, project management practice, Software Process Improvement (SPI) and relevant aspects of organizational theory.

\section{Fear}

A key finding from each of the independent studies was the negative impact that fear had on the successful operation of the respective virtual software teams [13]-[15], [9], [11], [17], [19]. Similar findings emerged from another independent study undertaken in Finland, which was presented and published at the International Conference on Global Software Engineering 2009 [61]. The authors of that paper acknowledge the relevance and corroborate the findings of the studies outlined here. They go on to state their findings, in many ways strengthen the conclusions about the origin and consequences of fear in GSD projects which have appeared in the authors published works on this topic [61].

The findings from each study with respect to fear are briefly presented here in summary form.

\subsection{Local Offsite Software Development}

The first study focused on local offsite software development. During the initial iteration of the action research cycle a number of key factors, which ultimately contributed to the failure of the local offsite strategy was identified. These included communication, cooperation, knowledge transfer, motivational and trust related problems, but the extent and seriousness of these issues were not obvious. Measures were taken to try to address these problems, but they had very limited success [7]. It emerged later that during this phase of the research project there was reluctance on the part of the offsite engineers to provide full information about what was actually going on and how they were being treated. This only came to light when further iterations of the action research cycle were undertaken.

At that point it was noted, the cost advantage the offsite engineers could provide had been frequently highlighted by management. This was in contrast to the requirement for the onsite engineers to be more productive and value adding to justify their higher salaries. This point was frequently reiterated and used to justify holding salaries at their current level and to help increase productivity of the onsite engineers. In these circumstances it was not surprising to discover it was totally counterproductive. This was the first time the impact of what would subsequently become known as the cost and productivity mantra as discussed in section 1.2 was encountered. A significant finding from this study was the level of fear, the implementation of the local offsite strategy had generated. In particular, the negative and serious impact this had on the motivation of the onsite engineers who were directly responsible for sending the work offsite and supporting the effort.

It also became clear this directly impacted on the other factors identified. The failure of management to recognize this fact, had a direct affect on the operation of the virtual teams and ultimately contributed to the overall failure of the strategy. It is relevant to note the full extent of the level of non-cooperation, the severity of the communication problems and lack of trust between the offsite and onsite engineers did not clearly emerge until the later iterations of the action research cycle [17].

\subsection{Offshore I Nearshore Software Development}

The second study investigated offshore / nearshore software development. An extensive investigation commenced to deal with the problems which were endangering the contract between Stock Exchange Trading Inc. and the Irish division of Software Future Technologies. It emerged during the initial iteration of the action research cycle that people who had worked very successfully together while collocated were now actively obstructing and blaming each other for all the problems that arose on their projects. This outcome was difficult to explain given the level of harmony achieved in their earlier collocated projects. The results from the initial investigation identified communication as the most pressing problem and the lack of trust, cooperation, cultural and process related problems as contributing factors. To address the issue of communication and the misuse of email to attack remote colleagues in particular a comprehensive communication policy was drawn up and implemented. While this addressed an important aspect of the problem it did not explain why this and other problems should have only arisen when the virtual team members were remotely located.

Further iterations of the action research cycle were undertaken. This resulted in motivation, the level of fear and the mistrust experienced by Stock Exchange Trading's virtual team members being identified as major contributing factors to the problems experienced. It emerged that while the majority of the teams were collocated in the US Stock Exchange Trading's virtual team members did not realize the full implications of the virtual team strategy. Once the virtual teams were established the possible impact on their day-to-day work, promotion and future employment prospects became clear. Management reinforced these negative aspects by utilizing the strategy to justify 
maintaining salaries at their existing levels. They also stressed the additional cost of US based staff and the need for them to be value adding to justify the extra expense which they incurred. As in the local offsite software development study the use of the cost and productivity mantra had serious implications for the operation of the virtual teams [13].

The outcome of this negative approach was individuals who genuinely feared that their jobs would be lost. This manifested itself in a lack of cooperation, mistrust, alienation and on occasions outright obstruction when and where the opportunities arose [14]. This was met with a similar negative reaction from the Irish team members who did not understand why they were the recipients of such hostile and aggressive behavior from people who they had previously successfully worked with. Fear and the impact it had on motivating those located in the organization from which the work was outsourced was identified as a major contributing factor to the problems experienced. The substantial negative contribution this made to the other factors the research identified was also recognized.

Once the negative impact fear played was identified, measures were taken to make it clear that there was no threat to the future of Stock Exchange Trading's engineers as a result of working with their Irish based colleagues. Indeed the partnership provided long term job security as it facilitated software maintenance and development at a competitive price and to the required level of quality by leveraging the advantages offered by both locations. The only serious threat to job security was presented by the failure of the virtual team strategy. If that happened the only viable alternative was to outsource the whole operation to Eastern Europe, the Far East or Latin America. The recognition of this fact, along with measures to address specific fear related issues helped tackle the problems which were experienced. This was coupled with the introduction of a comprehensive communication policy, cultural training, up skilling and an inclusive approach to process improvement, which led to the establishment of a productive working environment between staff at both locations. Cooperation and trust was reestablished between the virtual team members and the contract was successfully completed [13]-[14]. A more detailed discussion on how this was achieved is presented in section 7

\subsection{Offshore Software Testing}

The third study, offshore software testing focused on the operation of virtual testing teams with members based in Ireland (the location where the work was offshored from) and Malaysia. Both sites were divisions of the same multinational organization Computing World Inc. A Yin based case study and a sophisticated grounded theory approach to data gathering and analysis were employed. The data generated from this study was analyzed and resulted in the identification of one hundred and twenty eight initial concepts. These were further refined and incorporated into forty nine intermediate categories. The intermediate categories were evaluated further which resulted in the identification of four key factors. These consisted of three high level categories project management, communication, culture and a central category fear. The four key factors were broad enough to incorporate the intermediate categories. A graphical representation of the data gathering and analysis process implemented in this study is outlined in figure 2 .

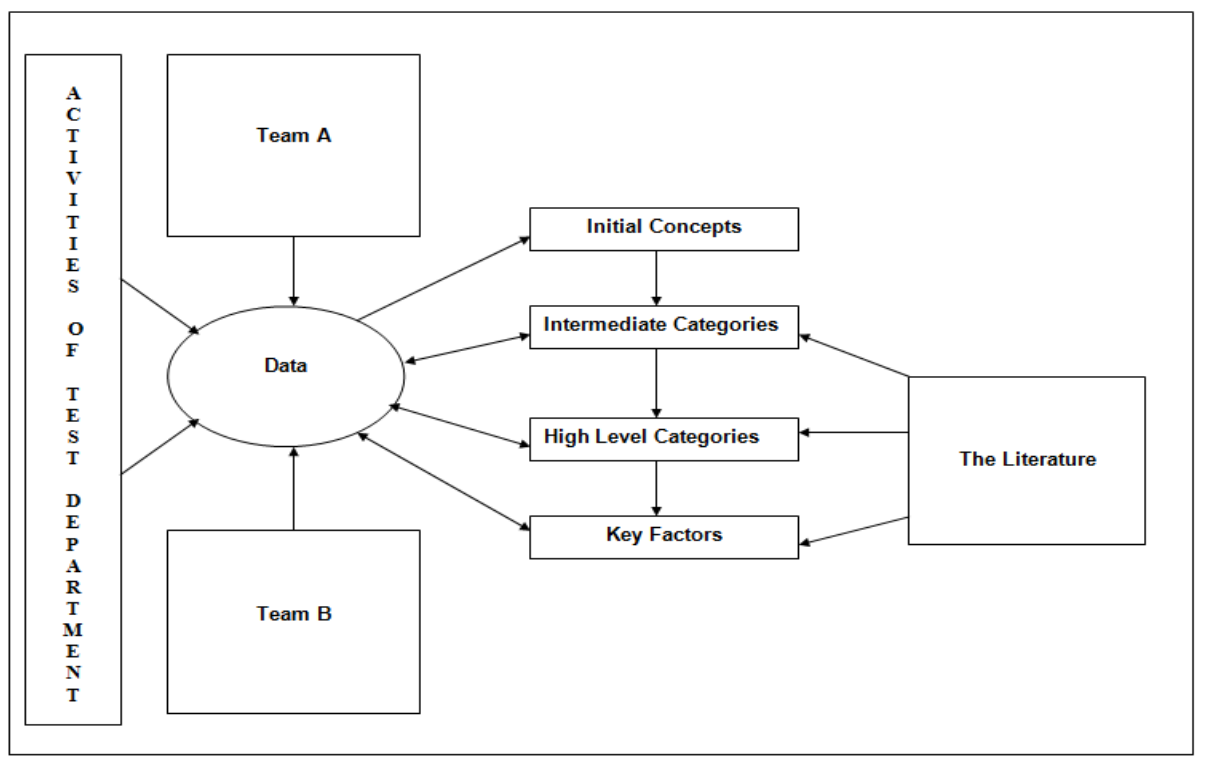

Figure 2: Overview of the Data Gathering and Analysis Process [19]

The central category to emerge from this study was the sustained level of fear of jobs being lost to Malaysia and the negative impact this had on the operation of the respective virtual teams. The part fear played emerged at an early stage of the investigation. As the study continued this was explored and further evaluated by observational 
research. Given the inductive nature of this study it was important that these findings were investigated further and reinforced by emerging directly from the analysis of the data which included interviews, questionnaires and focus groups. Even then alternative explanations were explored, but they only reinforced the previous findings. The identification of fear and its selection as the central category was based on the evidence of the significant impact it had on the operation of the virtual software testing teams. Instances of its effects were numerous and of a substantial and serious nature. While it had significant independent repercussions for the operation of both teams, it also impacted directly on important aspects of the other three high-level categories identified by this investigation which have been outlined in detail in [9], [16], [19].

Of particular relevance was how fear prevented the development of trust and cooperation [19] and how online communication was utilized to hinder the development of effective working relationships between virtual team members [9]. It also obstructed knowledge sharing and the provision of direct support between both locations. This took place in the context of the Irish and corporate management both regularly reinforcing the fact that the Malaysian engineer cost on average two fifths the salary of a comparable engineer in Ireland. This was used in an effort to motivate staff and as a mechanism to increase productivity. Just as in the two previous studies the use of the cost and productivity mantra was totally counterproductive and had a very negative impact as it demotivated the Irish staff. It also added to the level of fear and mistrust between the Irish and Malaysian virtual team members [17].

The Irish team members and managers lack of knowledge of the culture of their Malaysian colleagues also led to misunderstandings that reinforced the level of fear and mistrust which they experienced. In this context power distance clearly emerged from this study as a relevant issue [69]. The Malaysian staff demonstrated an unquestioning attitude to authority [75]. This resulted in their perceived agreement with everything that was said, not expressing an opinion, providing very little feed and saying yes to every management request. As one Irish team member stated: "I would say they (the Malaysians) are playing politics with people...I would say how they manage their part of the team over there, there is a lot going on we are not being made aware of... Would they actively be trying to undermine us? ... they would not be saying they were doing it" [8]. In these circumstances it was not surprising that cultural differences added to the overall levels of concern felt by Irish team members for their future. Fear and its influence on trust, communication, knowledge sharing and cooperation all negatively impacted on the operation of the virtual teams and their projects. These problems had serious implications for the success of the projects and the offshoring strategy [8].

\subsection{Offshore Software Development}

The fourth study focused on offshore software development and was an investigation into the experience of Indian managers who were responsible for managing software development projects which were outsourced to them. This research was undertaken with three Indian organizations. As a result of the inductive nature of the research the findings were structured under three main headings which emerged from the analysis of the data. These headings were team selection and establishment, team operation and the level of attrition. The team selection section focused on the key factors and issues the research identified which were relevant for the selection of Indian based software development teams and how these teams were effectively established. The team operation section considered dealing with GSD distance and the use of communication tools. It also incorporated the experience of Indian based Project Managers dealing with Western colleagues and clients. The level of attrition section focused on how these software organizations based in India held on to their existing staff in what is a very volatile labor market.

Of particular relevance was four out of the five respondents had experienced some level of sustained uncooperative and aggressive behavior from their remote colleagues and peers. This negatively impacted on communication, cooperation, trust and the overall performance of their projects. The experiences outlined were very similar to those highlighted in the other three studies which arose directly from fear that jobs would be lost to remote virtual team members and locations [11].

\section{Communication}

Effective communication is a vital process in every organization [27] and is an essential element for successful globally distributed software development [57]. Trust fear and motivation directly impact on the level, content, and effectiveness of communication and the use made of communication tools. Individuals have to be motivated to use the tools which are provided and these must be adequate to efficiently do the job. As a result the level of communication which takes place, it must be adequate to meet the needs of the specific teams and projects.

In the local offsite software development study the onsite engineers' part of whose work was sent offsite were only motivated to communicate with their offsite colleagues in a very limited manner. As a result communication was kept to a minimum, telephone calls were not returned, emails only provided a limited amount of information and on occasions were not responded to and queries remained unanswered. When direct communication took place the discourse was curt and on occasions aggressive. This resulted in inexperienced team members in an offsite location who lacked the communication, support and knowledge transfer they so badly needed to carry out their work successfully. It became clear online communication was being utilized as a means to limit and control the amount and quality of information which was shared. It was also being used as a barrier in the development of personal 
relationships and trust by limiting any direct interaction. It is very easy to resent and dislike someone you do not know personally, particularly when they are perceived as a serious threat to your future [9].

In the offshore / nearshore software development study, communication was used as a weapon with which to attack remote colleagues. This was primarily achieved thru the misuse of e-mail. The use of online communication for this purpose was relevant. The simple act of copying others on an email in itself is not necessarily a problem. Rather in this instance it was the premeditated and malicious use of this facility by virtual team members at both locations which facilitated it becoming a very serious issue. Managements' reaction to this problem in both organizations was also very relevant and the problem should have been identified and addressed at an early stage rather than being allowed to escalate. This study highlighted the necessity of having an effective communication policy in place to prevent or address this type of problem [13]. It also clearly demonstrated how communication tools could be misused to undermine and destroy trust in a virtual software team environment.

The offshore software testing study highlighted the use of email as a method of limiting information, knowledge transfer and personal contact, between the Irish and Malaysian virtual team members. This negatively impacted on the development of cooperation and trust which had serious repercussions for the effective operation of the virtual teams. An additional negative aspect to arise from the over reliance on the use of email was that issues normally took at least a day to be addressed. As there was an overlap in working hours between sites this was not utilized for synchronous communication. An important point to emerge from this study was that it is not enough to just provide communication tools, staff have to be trained and motivated to use them effectively. There was also a clear requirement for the implementation of a communication policy to help address these issues.

Training was provided for the Malaysian staff utilizing NetMeeting and conference calls. NetMeeting was a Microsoft product which offered basic video conferencing facilities which allowed PowerPoint presentations to be broadcast over the Internet. This approach to training proved unsuccessful and had to be abandoned. Without visual contact or knowledge of the technical and linguistic capabilities of participants, it was very difficult for the trainers to prepare material and assess how their presentations were received and understood in Malaysia. In addition a particular problem was that very little feedback was provided which proved to be a cultural issue [8]. The lack of face to face contact was identified as a serious problem and to address this, the trainers travelled to Malaysia to provide relevant courses. A number of key personnel from the Malaysian teams also travelled to Ireland for training. Both approaches provided better results than the online training strategy which had been initially implemented.

It also emerged from this study that there was a requirement for the adoption of a documented common vocabulary between sites. The experience level of some of the remote team members was very limited and included a large number of graduates. In these circumstances it should have been anticipated that they would not be familiar with the specific organizational terminology for artifacts, deliverables, procedures, steps, practices and acronyms. This problem was finally addressed by the establishment of a group of virtual team members focused on the development of a common vocabulary. The group consisted of four Irish members and four Malaysian members. The Irish members, based on their experience identified the terminology that they considered would be relevant. This list was sent to the Malaysians members for review. The list was reviewed amended and returned to the Irish members who wrote clear and concise definitions for the terms identified. These definitions were then reviewed by the Malaysian members and amended as required to add clarity and remove any possibility of ambiguity. Finally each definition was reviewed by a joint meeting of both the Irish and Malaysian group members, which took play by teleconference. Only when total agreement was achieved on each definition, was it accepted as part of the common vocabulary and published.

The results from the offshore software development study highlighted the importance that communication played in the successful operation of virtual software teams. The requirement for the selection of adequate communication tools was highlighted as was the importance of providing training in their use [18]. The need for a comprehensive communication procedure was identified. The importance of utilizing synchronous rather than asynchronous communication tools was recognized. It was also noted that the excessive use of email could lead to delays.

An interesting finding to emerge from the offshore software development study was in the Indian organizations where this research took place Instant Messenger was not used. It was seen as a possible security risk and was avoided in case of misuse. This is in contrast to its popularity and use as an effective communication tool when operating in a GSD environment [4], [21], [40]. In this situation Instant Messenger is normally utilized as a tool to facilitate informal discussion between remote colleagues [39].

\section{Trust}

Trust in the organizational setting is defined as the "reciprocal faith in others intentions and behavior. In short we give what we get: trust begets trust, distrust begets distrust" [49]. The importance of trust has become increasingly recognized as a critical element in the successful operation of organizations and specifically of business, professional and employment relationships [51]. Trust is the foundation of successful cooperation amongst individuals within and between organizations. It is essential for the functioning of an organization and the units 
operating within it. High levels of trust within an organization improves performance, efficiency, productivity, creativity and the overall results achieved [46].

In collocated software teams trust takes time to build between team members and is recognized as an important factor for success. The addition of distance makes it more difficult to establish trust between remote colleagues [5]. Despite this, the development of trust is essential for successful virtual team based software development [67]. The importance of this point is reinforced by the following statements:

- "Webs of technology and trust link virtual teams" [53]

- "Trust is pivotal in a global virtual team to reduce the high level of uncertainty endemic to the global and technologically based environment" [44].

In these circumstances it is important to recognize that the lack of trust has been identified as an important consequence of globally distributed software development [5], [24], [41], [60]. Therefore the establishment of trust in a globally distributed team environment is an important objective.

Cooperation between team members is essential for the successful operation of virtual teams. The term "teamness" [5] has been broadly defined as the ability of individuals to collectively collaborate and work effectively as a team [34]. Carmel (1999) has identified the loss of teamness as one of the five negative centrifugal forces which results from virtual team operation [5]. Distance has a negative impact on the level of teamness between remote colleagues [40], coupled with the fact that it is not easy to successfully integrate geographically remote and culturally diverse individuals or groups into a single team [3]. Add the impact of fear and it is easy to understand why in these circumstances problems can and do arise.

In the virtual team environment to facilitate collaboration and cooperation there is a specific requirement for trust to be developed [65], [69]. Trust is established through getting to know and building relationships between individual team members. When this is successfully achieved the results can be a motivated and cohesive team with a common purpose and shared goals and objectives. While this is the intention the reality is that the GSD environment directly militates against the establishment of trust.

Fear by its very nature undermines and inhibits the development of cooperation, trust and teamness. It is very difficult for individuals and groups to trust and build relationships with people who they fear are ultimately going to take their jobs. This was highlighted by the local offsite software development study where the onsite engineers mistrusted the offsite engineers and saw them as a potential threat to their future employment. In this instance trust was never established. This resulted in communication problems, limited knowledge transfer, uncooperative behavior, on occasions outright obstruction and ultimately the failure of the local offsite strategy [12], [17].

Even where trust and good relationships had previously existed as in the offshore / nearshore software development study they soon broke down under the pressure of this type of fear. A key element in that breakdown was the loss of trust between staff in both locations. This resulted in uncooperative behavior which saw email being used to attack each other. Every opportunity was taken to obstruct and highlight remote colleagues in a negative light. Rather than working as single teams, the virtual team members on all the teams were working to discredit each other based on geographical location. As a result project milestones were not met, budgets were exceeded and the projects were delivered late. There were serious problems regarding the quality of the software products that were delivered. If corrective action had not been taken this would have resulted in the failure of the strategy and the cancellation of a multimillion dollar contract.

In the offshore testing case study the lack of trust had serious implications for the success of the virtual team strategy. It is very difficult to trust someone you don't know. The development of trust was further inhibited as people feared they might lose their jobs to their remote colleagues. This resulted in the Irish team members endeavoring to limit their direct contact with their Malaysian colleagues. This lead to an over reliance on the use of email and it was the primary method of communication between remote team members. The Irish staff were actively encouraged to use the telephone and Instant Messenger, but they consistently made excuses and refused to do so. The reasons stated for their refusal were they did not know if they were contacting a man or a woman, or what order their remote colleagues first and surnames were in. The question why gender and / or the order of a person's name should prevent direct contact or why these issues had not been addressed were not satisfactorily explained. Even though, these questions were asked on numerous occasions. When the use of the organization's intranet was suggested as a location where team member's pictures, names and correct form of address from both sites could be displayed, the Irish staff rejected this suggestion. Clearly they were not motivated to use the range of communication tools provided. When they did communicate it emerged there was a general reluctance to provide information to their remote colleagues due to the level of fear they felt at the possibility of losing their jobs to the Malaysian division [9].

In the offshore software development study the lack of trust between the staff at the outsourcing and those at the remote locations were highlighted as a serious problem. This manifested itself in a number of ways which included the need to record telephone conversations and save emails to insure that information which had been provided from the outsourcing locations could be validated if and when required. This practice was also seen as of a method of 
discouraging the communication of limited, misleading or inaccurate information. The fact that these measures had to be employed and were widely used, indicate how prevalent these types of problems had been.

The very structure of distributed software development facilitates the identification of remote colleagues as a common enemy [17]. Ironically a key motivator for a group is to have a common enemy [36]. In the four studies outlined here to a greater or lesser extent a clear "them and us" culture existed [60] between the virtual team members based on their geographical location. The reality was personal relationships between sites did not exist or had seriously broken down. Fear and the lack of trust negatively impacted on the building of effective cross-site relationships and cohesive teams. This resulted in clear examples of not wanting to cooperate and share knowledge with remote colleagues. All of these issues are key factors for successful project management and had serious implications for the success of all the respective projects researched.

\section{How Trust was Reestablished and Developed}

The second study focused on the establishment of offshore / nearshore virtual teams by US based Stock Exchange Trading Inc. and the Irish division of Software Future Technologies. As outlined in section 4.2 when the fears of the US based virtual team members of their jobs being lost to their Irish colleagues was identified, a number of measures were put in place. The first was an agreement was reached with the management of Stock Exchange Trading Inc. that they would no longer use the cost and productivity mantra. The use of this approach had clearly failed and proved to be divisive and counterproductive. There was a need for open and frank communication between the relevant management and staff of both organizations. It was important that the long term strategy was clear and the benefits it offered to both companies and their staff were articulated. This took place and the benefits of the strategy and the alternatives if it failed were presented and discussed. Having addressed the issue of fear it was considered important to reestablish cooperation and trust between the team members. The objective was to maximize the potential benefits the virtual team strategy could provide [14].

Given the geographical distance and the limited opportunities for face to face contact the use of online communication tools were seen as key for the development and maintenance of trust between the virtual team members. Having addressed the problem of the misuse of email it was now utilized as a mechanism for collaboration and information exchange. Training on how to communicate effectively in the virtual team environment was provided to team members in both locations. It was recognized that personal contact and the development of cohesive and collaborative teams was necessary and a priority. To this end Instant Messenger was utilized to facilitate informal contact and its use was actively encouraged. This proved very successful and helped to restore and develop working relationships and reestablish trust between US and Irish based virtual team members.

Intranet sites were developed for each team to facilitate information sharing. Each team had its own pages which contained photographs and information about team members and their projects. It was also where project specific process documentation and templates were stored and accessed. Knowledge sharing was encouraged and bonuses were offered to those who contributed relevant information. The intranet was utilized to store and share this information and relevant documents and presentations were deposited in a knowledge base which all the teams had access to. Video conferences rather than telephone conferences were used to maximize face to face contact. It was considered important that at each meeting a few minutes were allowed for informal conversation and discussion.

A number of bespoke online tools were developed to facilitate configuration management and document exchange and approval. These proved very successful. The tools were developed in a collaborative manner with staff at both locations providing suggestions as to what they should contain and when relevant these were incorporated into the applications. In this way joint ownership of the tools was reinforced and suggestions for improvement were encouraged for inclusion in future versions.

The project managers played a key role in the development of trust. Every opportunity was utilized to develop collaborative and supportive teams. The project manager was responsible for insuring that the objectives of the offshore / nearshore strategy were clearly understood by all their team members in both locations. Communication was actively encouraged and monitored between team members across both sites. The goal was the development of professional relationships which would facilitate the reestablishment of trust between the virtual team members. The project managers saw this as a key objective and basis on which to build effective and collaborative virtual teams.

The performance of each team was evaluated as a single unit and not based on geographical location. This helped to reinforce a unified team approach and provided common goals, objectives and rewards. Within a short period of time teams with members who had been actively undermining each other were now communicating, cooperating and operating as unified teams, which delivered projects on time and within budget. It was agreed by both organizations that the virtual team strategy was now a success and the contract was successfully completed. The importance that the reestablishment and development of trust played was identified as a key factor in the achievement of that success. 
The contract was not renewed with the Irish division of Software Future Technologies. In the negotiations of the second contract, cost proved to be the deciding factor. Ireland was no longer a low cost location for software development and the focus of the Irish software industry was now knowledge based, niche centric and value adding. Therefore the Irish based Software Future Technologies was unable to compete on price and the contract went to India. It is relevant to note that Stock Exchange Trading Inc. continued to successfully utilize the modified virtual team strategy which this research made a substantial contribution in developing, with its new outsourcing partner [13].

\section{Implications}

Having undertaken these four independent studies a totally unexpected outcome was the commonality of the findings which emerged. The research methodologies employed, ensured that every effort was made to prevent the findings from the previous studies influencing the outcome of those subsequently undertaken. This resulted in the identification of a number of GSD key factors which need to be addressed to facilitate the establishment and effective operation of virtual software teams [10]. As a result of this research it was clear that at the organizational level there was a need for guidance on how to implement a virtual team GSD strategy. Based on the results from these studies and to address this the "GSD Implementation Model" has been developed [20].

The GSD Implementation Model provides a process through which organisations can approach the implementation of a GSD strategy. It is practical and comprehensive in its structured and iterative approach. Within its five phases it addresses the specific requirements of operating in a GSD virtual team environment based on the results from these studies. This is achieved by ensuring the rationale for undertaking a GSD strategy is clearly articulated and understood and that realistic objectives and goals are set. Senior management support is secured on achievable expectations based on the accurate evaluation of costs and risks. The required infrastructure, processes and supports are put in place to facilitate the operation of the virtual teams. Time and effort is put into effectively establishing and managing the teams. This includes addressing fear, facilitating communication and developing trust. An effective project management strategy based on the needs of the GSD environment is implemented. Key to the long term success of this approach is the documenting and leveraging of the experience gained implementing such a strategy [18].

It was also recognized at an industry wide level that software process improvement maturity models were very limited in what they provided to deal with the specific issues, which are relevant to GSD projects and virtual team operation. To address this, work has been undertaken based on the results from these studies to develop an additional process area called "Global Teaming" [64] for the Capability Maturity Model Integration ${ }^{\circledR}\left(\mathrm{CMMI}^{\circledR}\right)$ [23]. The goal of this process area is to specifically address the requirements of developing trust and teamness in the CMMI ${ }^{\circledR}$ global software engineering environment. The author is also actively involved in the development of a software process improvement model for the Medical Device Industry, called Medi SPICE [54] which is based on ISO 155045 [43]. This model will incorporate process areas that are specific to the requirements of operating in a global software distributed environment. These processes are currently being developed [55] and are based on the findings from these studies. The development of trust in the GSD and virtual team setting will be a key part of the GSD aspect of the Medi SPICE model.

\section{Conclusion}

Globally distributed software development and in particular the implementation of a virtual team based strategy is a difficult and complex endeavor. This has been discovered by many organizations that have implemented this approach and is highlighted from the results of the studies outlined in this paper. The social aspects of professional team based software development are very important and are often overlooked or not addressed. This is particularly relevant in a globally distributed virtual team environment

When implementing such a strategy organizations must recognize that virtual teams operate differently to collocated teams, therefore they must be managed differently. These differences arise due to the complex and collaborative nature of software development and the impact distance introduces. In these circumstances a key problem is the establishment and maintenance of trust. This paper has focused on identifying this problem and outlining practical measures which have been successfully implemented to address it. Trust does not just occur, particularly in the virtual software team environment. It has to be actively facilitated, fostered and developed. Knowledge of the factors which prevent, undermine and facilitate trust is key to the success of every GSD and virtual software team strategy.

\section{Acknowledgment}

This research is supported by the Science Foundation Ireland (SFI) Stokes Lectureship Programme, grant number 07/SK/I1299 and the SFI Principal Investigator Programme, grant number 08/IN.1/I2030 


\section{References}

[1] R. L. Baskerville, Distinguishing action research from participative case studies, Journal of Systems and Information Technology, vol. 1, no. 1, pp. 25-45, 1997.

[2] R. L. Baskerville, Investigating information systems with action research, Communications of the Association for Information Systems, vol. 2, no. 1, pp. Article 19, 1999.

[3] R. D. Battin, R. Crocker, J. Kreidler, and K. Subramanian, Leveraging resources in global software development, IEEE Software, vol. 18, no. 2, pp. 70-77, 2001.

[4] D. Boland, and B. Fitzgerald, Transitioning from a co-located to a globally-distributed software development Team: A Case Study at Analog Devices Inc., in Proceedings of the International Conference on Software Engineering (ICSE 2004), Edinburgh, Scotland, 2004.

[5] E. Carmel, Global Software Teams: Collaboration Across Borders and Time Zones. Saddle River, NJ: Prentice Hall, 1999.

[6] E. Carmel and P. Tjia, Offshoring Information Technology: Sourcing and Outsourcing to a Global Workforce. Cambridge, UK: Cambridge University Press, 2005.

[7] V. Casey, The application of a CMM based process improvement initiative to a remotely located tool based software application development environment, vol. MSc.: University of Limerick, Ireland, 2000.

[8] V. Casey, Leveraging or Exploiting Cultural Difference?, in Proceedings of the 4th IEEE International Conference on Global Software Engineering (ICGSE). Limerick: IEEE, 2009.

[9] V. Casey, Software Testing and Global Industry: Future Paradigms. Newcastle, UK: Cambridge Scholars Publishing, 2009.

[10] V. Casey, Virtual software team project management, Journal of the Brazilian Computer Society, vol. 16, no. 2, 2010.

[11] V. Casey, S. Despande, and I. Richardson, Outsourcing Software Development The Remote Project Manager's Perspective, in Proceedings of the 2nd Information Systems Workshop on Global Sourcing, Services, Knowledge and Innovation, Val d'Isére, France, 2008.

[12] V. Casey and I. Richardson, A practical application of the IDEAL model, Software Process Improvement and Practice, vol. 9, no. 3, pp. 123-132, 2004.

[13] V. Casey and I. Richardson, Practical experience of virtual team software development, in Proceedings of European Software Process Improvement (Euro SPI), Trondheim, Norway, 2004.

[14] V. Casey and I. Richardson, Virtual software teams: Overcoming the obstacles, in Proceedings of the 3rd World Congress for Software Quality, Munich, Germany, 2005.

[15] V. Casey and I. Richardson, Project management within virtual software teams, in Proceedings of the 1st IEEE International Conference on Global Software Engineering, Florianopolis, Brazil, 2006.

[16] V. Casey and I. Richardson, Uncovering the reality within virtual software teams, in Proceedings of 1st International Workshop on Global Software Development for the Practitioner, Shanghai, China, 2006.

[17] V. Casey and I. Richardson, The impact of fear on the operation of virtual teams, in Proceedings of the 3rd IEEE International Conference on Global Software Engineering, Bangalore, India, 2008.

[18] V. Casey and I. Richardson, A structured approach to global software development, in Proceedings of European Systems and Software Process Improvement and Innovation, Dublin, Ireland, 2008.

[19] V. Casey and I. Richardson, Virtual teams: Understanding the impact of fear, Software Process Improvement and Practice, vol. 13, no. 2, pp. 511-526, 2008.

[20] V. Casey and I. Richardson, Implementation of global software development: A structured approach, Software Process Improvement and Practice, vol. 14, no. 5, pp. 247-262, 2009.

[21] J. Chisan and D. Damian, Towards a Model of Awareness Support of Software Development in GSD., in Proceedings of the International Conference on Software Engineering (ICSE 2004). Edinburgh, Scotland, 2004.

[22] V. Clerc, P. Lago, and H. van Vliet, Global Software Development: Are Architectural Rules the Answer?, in Proceedings of the 2nd IEEE International Conference on Global Software Engineering ICGSE 2007, Munich, 2007.

[23] CMMI® Product Team, Capability Maturity Model® Integration for Development, Software Engineering Institute, Pittsburgh, PA, USA 2006.

[24] D. Damian and D. Zowghi, Requirments engineering challenges in multi-site software development organizations, Requirements Engineering, vol. 8, pp. 149-160, 2003.

[25] W. H. Davidow and M. S. Malone, The Virtual Corporation. New York: Edward Brulingame Books/Harper Business, 1992

[26] E. J. Davidson and A. S. M. Tay, Studying Teamwork in Global IT Support, in Proceedings of the 36th Hawaii International Conference on Systems Science, Kona-Kailua, HI, 2003.

[27] S. Dawson, Analysing Organisations. London, UK: The Macmilland Press Ltd, 1992.

[28] G. DeSanctis, N. Staudenmayer, and S. S. Wong, Interdependence in Virtual Organizations, vol. 6. Chichester: John Wiley \& Sons., 1999.

[29] S. Deshpande and I. Richardson, Management at the Outsourcing Destination - Global Software Development in India, in Proceedings of the 4th IEEE International Conference on Global Software Engineering (ICGSE), Limerick, Ireland, 2009.

[30] S. Deshpande, I. Richardson, V. Casey, and S. Beecham, Culture in Global Software development - a Weakness or Strength?, in Proceedings of the 5th International Conference on Global Software Engineering (ICGSE 2010), Princeton, NJ, USA, 2010.

[31] C. Ebert and P. De Neve, Surviving Global Software Development, IEEE Software, vol. 18, pp. 62 - 69, 2001. 
[32] M. Elden and R. F. Chisholm, Emerging Varieties of Action Research: Introduction to the Special Issue, Human Relations, vol. 46, no. 2, pp. 121-142, 1993.

[33] J. Gill and P. Johnson, Research Methods for Managers. London UK, 1997.

[34] A. Glaser, S. Fu, and M. Tumelty, On Site: Growing A Participatory Programming Environment, Communications of the ACM, vol. 47, no. 2, pp. 27-29, 2004.

[35] C. Handy, Trust and the Virtual Organization, Harvard Business Review, vol. 73, no. 3, pp. 40-50, 1995.

[36] C. B. Handy, Understating Organizations. London, UK: Penguin Books, 1985.

[37] I. S. Hayes, Ready or Not: Global Sourcing Is in Your IT Future, Cutter IT Journal, vol. 15, pp. 5-11, 2002.

[38] J. D. Herbsleb, Global Software Engineering: The Future of Socio-technical Coordination, in Proceedings of Software Engineering (FOSE'07), Minneapolis, MN, USA, 2007.

[39] J. D. Herbsleb, D. L. Atkins, D. G. Boyer, M. Handel, and T. A. Finholt, Introducing instant messaging and chat in the workplace, in the Proceedings of ACM Conference on Human Factors in Computing Systems, Minneapolis, MN, USA., 2002.

[40] J. D. Herbsleb and A. Mockus, An empirical study of speed and communication in globally distributed software development, IEEE Transactions on Software Engineering, vol. 29, no. 6, pp. 481-494, 2003.

[41] J. D. Herbsleb and D. Moitra, Global Software Development, IEEE Software, vol. 18, no. 2, pp. 16-20, 2001.

[42] M. Hertzum, The importance of trust in software engineers' assessment and choice of information sources, Information and Organization, vol. 12, no. 1, pp. 1-18, 2002.

[43] ISO/IEC 15504-5: 2006, Information Technology - Process Assessment - Part 5: An exemplar Process Assessment Model, JTC 1/SC 7

[44] S. L. Jarvenpaa and D. E. Lindner, Communication and trust in global virtual teams, Organization Science, vol. 10 , no. 6 , pp. $791-815,1999$

[45] M. Jensen, S. Menon, L. E. Mangset, and V. Dalberg, Managing Offshore Outsourcing of Knowledge-intensive Projects - A People Centric Approach, in Proceedings of International Conference on Global Software Engineering (ICGSE 2007), Munich, 2007.

[46] Y. Keyzerman, Trust in Virtual Teams, in Proceedings of IEEE International Professional Communication Conference, 2003, Orlando, Florida, USA., 2003.

[47] H. K. Klein and M. D. Myers, A set of principles for conducting and evaluating interpretive field studies in information systems, MIS Quarterly, vol. 23, no. 1, pp. 67-93, 1999.

[48] J. Kotlarsky and I. Oshri, Social Ties, Knowledge sharing and successful collaboration in globally distributed system development projects, European Journal of Information Systems, vol. 14, no. 1, pp. 37-48, 2005.

[49] R. Kreitner, A. Kinicki, and M. Buelens, Organizational Behavior, First European Edition ed. London UK: McGraw-Hill Publishing Company, 1999.

[50] A. Lamersdorf, M. Jürgen, and R. Dieter, A survey on the state of the practice in distributed software development: criteria for task allocation, in Proceedings of 4th IEEE International Conference on Global Software Engineering (ICGSE) 2009, Limerick, Ireland, 2009.

[51] R. J. Lewicki and B. B. Bunker, Developing and mantaining trust in work relationships, in Trust In Organizations: Frontiers of Theory and Research. London UK: Sage Publications, 1996, pp. 114-139.

[52] J. Lipnack and J. Stamp, Virtual Teams: Reaching Across Space, Time And Originating With Technology: John Wiley \& Sons, 1997.

[53] J. Lipnack and J. Stamp, Virtual Teams: People Working Across Boundaries with Technology, 2nd ed. New York: John Wiley \& Sons. Inc, 2000.

[54] F. McCaffery and A. Dorling, Medi SPICE: An overview, in Proceedings of the International Conference on Software Process Improvement and Capability Determinations (SPICE), Turku, Finland, 2009.

[55] F. McCaffery, A. Dorling, and V. Casey, Medi SPICE: An Update, in Proceedings of the International Conference on Software Process Improvement and Capability Determinations (SPICE), Pisa, Italy, 2010

[56] N. B. Moe and D. Šmite, Understanding a lack of trust in global software teams: A multiple-case study, Software Process: Improvement and Practice, vol. 13, no. 3, pp. 217-231, 2008.

[57] B. A. Nardi, S. Whittaker, and E. Bradner, Interaction and Outeraction: Instant messaging in action, in Proceedings of ACM Conference on Computer Supported Cooperative Work, Philadelphia, Pennsylvania, United States, 2000

[58] J. A. O'Brien, Management Information Systems Managing Information Technology in the Business Enterprise, Sixth ed: Mc Graw Hill Irwin, 2002

[59] E. Ó Conchúir, P. J. Ågerfalk, H. H. Olsson, and B. Fitzgerald, Global software development: where are the benefits? Communications of the ACM, vol. 52, no. 8, pp. 127-131, 2009.

[60] H. L. Oppenheimer, Project management issues in globally distributed development, in Proceedings of ICSE Workshop on Global Software Development, 2002.

[61] A. Piri, T. Niinimäki, and C. Lassenius, Descriptive Analysis of Fear and Distrust in Early Phases of GSD Projects, in Proceedings of the 4th IEEE International Conference on Global Software Engineering. Limerick, Ireland, 2009.

[62] A. Powell, G. Piccoli, and B. Ives, Virtual teams: A review of current literature and direction for future research, The DATA BASE for Advances in Information Systems, vol. 35, no. 1, pp. 6-36, 2004.

[63] R. Prikladnicki, J. L. N. Audy, and R. Evaristo, Global software development in practice lessons learned, Software Process Improvement and Practice, vol. 8, pp. 267-279, 2003.

[64] I. Richardson, V. Casey, J. Burton, and F. McCaffery, Global Software Engineering: A Software Process Approach, in Collaborative Software Engineering, (I. Mistrík, J. Grundy, A. Hoek, and J. Whitehead, Eds.). Springer, 2010, pp. 35-56. 
[65] W. Royce, Software Project Management: A Unified Framework. Reading, Mass. USA: The Addison-Wesley, 1998.

[66] A. F. Rutkowski, D. R. Vogel, M. Van Genuchten, T. M. A. Bemelmans, and M. Favier, E-collaboration: The reality of virtuality, IEEE Transactions on Professional Communication, vol. 45, no. 4, pp. 219-230, 2002.

[67] R. Sabherwal, The role of trust in outsourced IS development projects, Communications of the ACM, vol. 42 , no. 2, pp. 80-86, 1999.

[68] S. Sahay, B. Nicholson, and S. Krishna, Global IT Outsourcing: Software Development across Borders. Cambridge UK: Cambridge University Press, 2003.

[69] S. C. Schneider and J.-L. Barsoux, Managing Across Cultures, 2nd ed. Harlow: Financial Times Prentice Hall, 2002.

[70] S. Slaughter and S. Ang, Employment Outsourcing In Information Systems, Communications Of The ACM, vol. 39, no. 7, pp. 47-54, 1996.

[71] A. Strauss and J. Corbin, Basics of Qualitative Research: Grounded Theory Procedures and Techniques, First ed. London: Sage Publications, 1990.

[72] A. Strauss and J. Corbin, Basics of Qualitative Research: Techniques and Procedures for Developing Grounded Theory, Second ed. Thousand Oaks, CA, USA: Sage Publications, 1998.

[73] G. Susman and R. Evered, An assessment of the scientific merits of action research, The Administrative Science Quarterly, vol. 23, no. 4, pp. 582-603, 1978.

[74] S. S. Toaff, Don't play with mouths of fire, and other lessons of global software development, Cutter IT Journal, vol. 15, no. 11, pp. 23-28, 2002.

[75] F. Trompenaars and C. M. Hampden-Turner, Riding the Waves of Culture: Understanding Cultural Diversity in Global Business, 2nd ed. New York, NY, USA: McGraw-Hill, 1998.

[76] R. K. Yin, Case study research / design and methods, vol. 5, Second ed. Thousand Oaks, CA. USA: Sage Publications, 1994. 